\title{
Analysis of mobile applications that help skin care through facial recognition: A systematic analysis
}

\section{Análisis de aplicaciones móviles que ayuden al cuidado de la piel mediante reconocimiento facial utilizando sistemas inteligentes. Un análisis sistemático}

\section{Caiza Caizabuano José Ruben, Mise Pasuña José Luis, Diego Fernando Andaluz Espinosa, and Rolando Patricio Reyes Chicango}

Universidad de las Fuerzas Armadas ESPE, Latacunga, Ecuador

\section{Abstract}

Corresponding Author: Caiza Caizabuano José Ruben jrcaiza@espe.edu.ec

Received: 24 December 2019 Accepted: 2 January 2020 Published: 8 January 2020

Publishing services provided by Knowledge E

(c) Caiza Caizabuano José Ruben et al. This article is distributed under the terms of the Creative Commons

Attribution License, which permits unrestricted use and redistribution provided that the original author and source are credited.

Selection and Peer-review under the responsibility of the SIIPRIN-CITEGC Conference Committee.
The knowledge and integration of new technologies has shown that it is possible to improve access to health services, such as skin care and diagnosis of pathologies in the area of dermatology, applying mobile technologies and ap- plications. In this systematic review it seeks to investigate mobile applications that use intelligent systems that help skin care through facial recognition. The investigation was carried out between 2010 and 2018, finding 168 articles that resemble the subject; that were reduced to 28 articles with available text, after a dissertion of articles that did not contribute to the research as well as repeated. Finally, three of them were chosen to begin the topic of study. The present review allows us to conclude that there is a guideline for using mobile applications and technologies for the care and diagnosis of pathologies on the skin by means of algorithms, models, techniques and methods applying intelligent systems. How- ever, the integration of mobile applications directly for skin care is just beginning the research field, but offers promising processes and results.

Resumen. El conocimiento e integración de nuevas tecnologías, ha demostrado que se puede mejorar el acceso a servicios de la salud, como es al cuidado de la piel y diagnóstico de patologías en el área de la dermatología, aplicando tecnologías y aplicaciones móviles. En esta revisión sistemática busca indagar aplicaciones móviles que usan sistemas inteligentes que ayuden al cuidado de la piel mediante reconocimiento facial. La indagación fue realizada entre 2010 y 2018, encontrando 168 artículos que se asemejan al tema; que se redujeron a 28 artículos con texto disponible, luego de una dicersion de artículos que no aportaban a la investigación como también repetidos. Finalmente, tres de ellos se eligió para empezar el tema de estudio. La presente revisión permite concluir que hay una directriz para usar aplicaciones y tecnologías móviles para el cuidado y el diagnostico de patologías sobre la piel mediante algoritmos, modelos, técnicas y métodos aplicando sistemas inteligentes. Sin embargo, la integración de aplicaciones móviles directamente para el 
cuidado de la piel, apenas inicia el campo de investigación, pero ofrece procesos y resultados prometedores.

Keywords: Mobile applications, Skin care, tele dermatology, mobile technolo- gies, systematic review.

Palabras clave: Aplicaciones móviles, Cuidado de la piel, tele dermatología, tecnologías móviles, revisión sistemática.

\section{Introducción}

La tecnología móvil ha evolucionado con gran velocidad [11] mostrando al mundo un potencial de mejorar la vida de los seres humanos. En hardware ha evolucionado con el uso de teléfonos inteligentes debido a su diseño y tamaño llegando a la miniaturización [2I]. En software se ha visto la rápida evolución de aplicaciones móviles que facilitan las tareas diarias de las personas. A la vez, se fabrican dispositivos móviles más sofisticados, esto ha permitido que las aplicaciones móviles desarrolladas aumenten su funcionalidad y productividad [3], un ejemplo de tecnología aplicada es realidad aumentada [4] y redes neuronales [5]. Existen infinidad de aplicaciones móviles que están inmersas en diferentes campos de conocimiento como son: empresarial, educativo, entretenimiento, videojuegos, salud, etc. Que ha permitido reforzar el proceso de generación del conocimiento [6], y alcanzar sus objetivos publicitarios.

La tecnología móvil, ha contribuido de manera positiva en el campo de la salud. Un ejemplo es la autogestión en la salud, es la aplicación MedsBox actúa como una aplicación para la autogestión del paciente para su propia ingesta de medicamentos [4]. Con el avance de tecnología móvil ha incrementado el desarrollo de aplicaciones móviles para la salud, podemos mencionar: fitness app, diabetes app, meditación app y cáncer app, han llegado a ser las categorías de mayor crecimiento [8]. Estas aplicaciones permiten saber cuántos pasos hemos dado al día, cuantas calorías se ha consumido, tomar la tensión arterial, cuando usar o no un protector solar.

Por ejemplo, las aplicaciones para la diabetes, como apoyo para toma de decisiones a los pacientes en su vida diaria usando un predictor de glucosa móvil, control de la glucosa, el autocuidado, alarmas sonoras y recordatorios para la administración oportuna de medicamentos [9--11]. Otros ejemplos de aplicaciones móviles para tratar 
la diabetes como: pie diabético, pues es un sistema de análisis de imágenes [12]. MSalud es aplicación de tecnología aplicada a la salud para pacientes con diabetes de tipo 2 [13], y sin dejar de lado las aplicaciones para la diabetes son más aceptadas por los jóvenes ya que permiten la conexión, autogestión, autoeducación, como ejemplo aplicando en la diabetes tipo 1 [14].

En esta revisión sistemática se describe aplicaciones móviles que ayuden al cuidado de la piel mediante reconocimiento facial utilizando sistemas inteligentes, para ello se realizó una lectura minuciosa y se obtuvo una detallada clasificación las aplicaciones móviles por destacar el de un sistema de tele dermatología móvil [34] cuyo análisis se basa en el rendimiento de las aplicaciones de teléfonos inteligentes en la evaluación del riesgo de melanoma.

Un aporte valioso para el cuidado de la piel es la CNN que usa algoritmo de la Red Neuronal Artificial, la aplicación de la inteligencia artificial que es capaz de clasificar el cáncer de piel con un diagnostico similar al de los dermatólogos [5].

Según datos que se obtuvo de una revisión sistemática [35], las enfermedades que más afectan a la piel es melanoma con el $75 \%$ de las muertes en todo el mundo. Para evitar este tipo de enfermedades y cuidar la piel, están realizando aplicaciones móviles que se pueden usar en los teléfonos inteligentes. Este tipo de aplicaciones son utilizadas por personas que viven en zonas remotas en países pobres en vías de desarrollo que no tienen acceso a un médico especialista. Las aplicaciones desarrolladas se basan en algoritmos de inteligencia artificial que analizan la imagen como benigna o maligna [12]. Para cuidar la piel del Melanoma, se han desarrollado varias aplicaciones móviles, como MED-NODE (sistema de soporte de decisión/experto) el sistema utiliza imágenes digitales no dermatoscopias de la piel, de donde se extrae automáticamente las regiones de las lesiones como el color y la textura [13]. Existen estudios sobre el melanoma y la detección de lesiones cutáneas utilizando una dermatoscopia digital de teléfono inteligente de aplicaciones donde se puede analizar imágenes de alta resolución de las lesiones de la piel y proporciona al usuario información sobre la apariencia de malignidad, también se estudia las aplicaciones móviles (teléfonos inteligentes), imágenes multiespectrales (MoleMate y MelaFi) [14, 15]. Adicional se ha considerado un sistema de soporte de gestión del cuidado de la piel basado en la computación en la nube, se propone un sistema de apoyo para la gestión del cuidado de la piel con un método de interacción, aprovechando un mecanismo de sincronización de datos permitiendo que los pacientes y el personal médico se visualicen simultáneamente $[16,17]$. 
Con la finalidad de acceder al cuidado de la piel desde cualquier lugar geográfico, se ha desarrollado una nueva tecnología en salud conocida como "tele dermatología", según estudios realizados permite a los pacientes de las zonas rurales acceder a un diagnóstico de dermatología donde comúnmente esta especialidad es administrada por personal de salud sin formación, para ello se ha desarrollado una aplicación móvil que permite analizar, diagnosticar y cuidar de la salud a distancia utilizando teléfonos inteligentes, la tele dermatología se puede aprovechar como una alternativa que agiliza los servicios de salud mediante la reducción de visitas innecesarias en persona hacia los dermatólogos, además se pueden usar como herramientas de capacitación de diagnóstico para médicos y dermatólogos, así también el cuidado del paciente [18-21]. De igual forma se pudo conocer la existencia de varias aplicaciones móviles que se encuentran alojadas en App Store y Google Play Store, mismas que ayudan al cuidado de la piel al indicar el índice UV de acuerdo con la ubicación actual del usuario [22], M-Skin es un sistema móvil que permite detección precoz del cáncer, este sistema utiliza técnicas de visión por computador y procesamiento de imágenes, para la segmentación se usa el algoritmo Grab Cut, y Support Vector Machine (SVM) es una técnica de clasificación en las características de la textura como área, perímetro, excentricidad de la piel [23].

Los trabajos antes mencionados, se han analizado las aplicaciones móviles que han permitido el cuidado de la piel del ser humano, es decir las formas más comunes para proteger la piel de diferentes enfermedades sea cáncer, melanoma, lesiones, diabetes, heridas e incluso como protegerse de los rayos UV. Los resultados previos muestran todas estas aplicaciones móviles, que para su desarrollo se enfocan procesos, modelos, métodos, herramientas y algoritmos de inteligencia artificial, sistemas inteligentes que son manipulados en un teléfono inteligente. En este artículo, se analiza el efecto de usar aplicaciones móviles para el cuidado de la piel mediante reconocimiento facial en sus diferentes especialidades.

\section{Métodos}

En la literatura se han encontrado varias revisiones sistemáticas donde se abordan temas relacionados que utilizan las aplicaciones móviles para el diagnóstico de enfermedades de la piel, la mayoría de ellas se centran únicamente en el cáncer de piel, sin embargo, hay que considerar que existen otro tipo de enfermedades dermatológicas que pueden ser problemáticas por sus efectos secundarios y conllevar a terapias muy 
graves, riesgosas y costosas que reducen considerablemente la calidad de vida del paciente.

En el contexto de este estudio, la población corresponde al conjunto de aplicaciones móviles de cuidado y diagnóstico de enfermedades dermatológicas, para lo cual, se determinaron las siguientes palabras clave:" apps" OR"mobile applications" OR "dermatology apps AND "smartphone".

\subsection{Criterios de Selección}

Se analizó, la incorporación de la tecnología móvil para el cuidado de la salud piel a distancia (tele dermatología). Después las aplicaciones móviles que permiten diagnosticar las enfermedades de la piel. Para finalizar se eligió las obras que hablan de aplicaciones que informan sobre el cuidado de la piel.

\subsubsection{Estrategia de Búsqueda}

Se realizó una búsqueda de en las bases de datos como: Scopus (71 publicaciones), Science Direct (72 publicaciones), IEEE Xplore Digital Library (25). El término utilizado para la búsqueda se relaciona con: "Skin care o cuidado de la piel" y "aplicaciones móviles o mobile application". La búsqueda se limitó de 2010-2018 con la siguiente cadena respectivamente:

Scopus (TITLE-ABS-KEY ("skin care" OR "skin surface "OR "skin texture") AND TITLEABS-KEY (mobile OR application AND mobile OR smartphones OR "cell phones") AND TITLE-ABS-KEY (process OR model OR method OR tool OR algorithm))

Sciente directy (TITLE-ABSTR-KEY ("skin care" OR "skin surface " OR "skin texture") AND TITLE-ABSTR-KEY (mobile OR application AND mobile OR smartphones OR "cell phones") AND TITLE-ABSTR-KEY (process OR model OR method OR tool OR algorithm)).

IEEE ((("skin care" OR "skin surface " OR "skin texture") AND mobile OR application AND mobile OR smartphones OR "cell phones") AND process OR model OR method OR tool OR algorithm

\subsubsection{Selección de Estudios}

En el proceso de búsqueda, se encontraron 11.347 artículos científicos entre 1947 y abril del 2018, que comprendían el término "cuidado de la piel". Por esta razón la búsqueda se limitó a los años de 2010-2018, se toma de referencia a este periodo por los últimos 


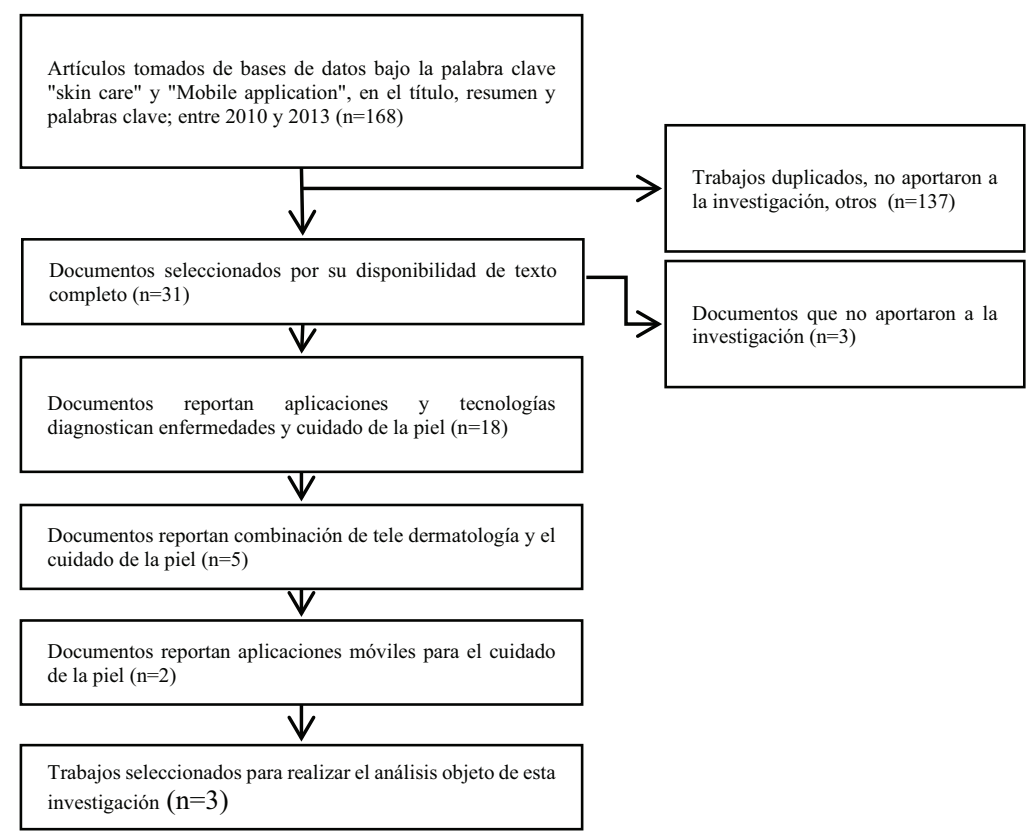

Figure 1: Flujo de análisis de fuentes para la investigación.

avances tecnológicos para el cuidado de la piel mediante aplicaciones. Se llevo a cabo la búsqueda con el termino en el título, resumen y las palabras claves, donde se obtuvo 168 artículos.

Consecuentemente, se eliminaron los artículos duplicados y los que no aportan a la revisión, reduciendo la muestra a 27 trabajos con disponibilidad de texto. Se utilizo la herramienta de gestión de referencias Zootero, para la clasificación de los trabajos de acuerdo a las tecnologías aplicadas al cuidado de la piel mediante aplicaciones móviles, para ello se realizó un análisis de la literatura disponible, agrupando por el tipo de tecnología aplicada. Posteriormente la muestra se redujo a 3 publicaciones que estudian al tema de investigación. El proceso de inclusión y exclusión de la revisión de la literatura podemos observar en la Fig. 1.

\subsection{Análisis de Datos}

La búsqueda de artículos se llevó a cabo, debido a la necesidad de establecer el estado del arte de las tecnologías o aplicaciones móviles aplicadas al cuidado de la piel, mediante sistemas inteligentes. La primera búsqueda se la realizo sin ninguna restricción utilizando el término cuidado de la piel, adquiriendo los resultados registrados en la sección anterior del presente trabajo. Posteriormente, se realizó tecnologías y aplicaciones móviles aplicadas, se clasifico de la siguiente manera: El 52\% son investigaciones son aplicaciones y tecnologías que diagnostican enfermedades de 
la piel, el 19\% específicamente hablan de la combinación de la tele dermatología como tecnología al cuidado de la piel, 11\% son documentos que reportan aplicaciones móviles para el cuidado de la piel, el 7\% de las obras no aportan con la investigación. Finalmente se encontró un $11 \%$ de los trabajos registrados en las bases de datos mencionadas aportan al cuidado de la piel mediante aplicaciones móviles desarrollados para sistemas operativos IO'S y Android [34].

\section{Resultados}

De 31 publicaciones referenciadas en el presente trabajo, 18 informan de sistemas que aplican tecnologías que diagnostican enfermedades de la piel, la mayor parte de referencias lo hacen mediante un algoritmos, métodos y herramientas [24, 25]; 5 documentos aportan a la tele dermatología como una tecnología para el diagnóstico y cuidado de la piel mediante asistencia remota, para el desarrollo de estos sistemas se han aplicado métodos y modelos ayudan al autocuidado de la piel [26], reconocimiento de lesiones [27]; 2 obras tratan de aplicaciones móviles desarrollada para el cuidado de la piel $[28,29]$ estos pueden analizar imágenes de la piel y dar un diagnóstico de las posibles patologías; y finalmente 3 obras tratan de aplicaciones móviles mismos que mediante un teléfono inteligente pueden notificar recomendaciones adecuadas para el cuidado de la piel en base al índice ultravioleta (UVI)[30], hay aplicaciones que desarrollan conductas de prevención del cáncer de piel [5], e incluyen aplicaciones asisten al cuidado de la piel de personas con espina bífida que son más propenso a lesiones cutáneas [31].

TABLE 1: Tecnologías que informan la aplicación de tecnologías para el cuidado de la piel.

Tipo de
tecnología
Aplicaciones y
Tecnologías
móviles
Tele
dermatología
Aplicaciones
móviles
Aplicaciones
seleccionadas
Aplicaciones que
no aportan
Total

$\begin{gathered}\text { Números de } \\ \text { publicaciones }\end{gathered}$
18
5
2
3
3
31

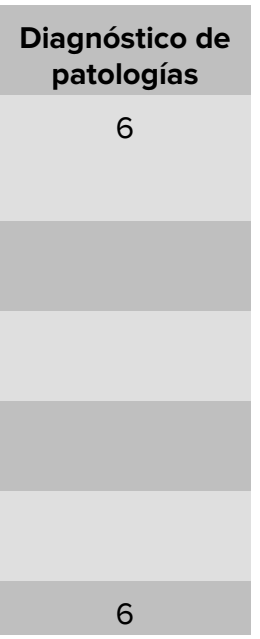


Este análisis incluye tres estudios que aportan al cuidado de la piel mediante aplicaciones móviles, utilizando métodos y modelos en el desarrollo de aplicaciones que permiten el cuidado de la piel mediante sistemas inteligentes.

\subsection{Descripción de estudios}

La CNN o Redes Neuronales profundas [5] demuestra una capacidad de la inteligencia artificial de clasificar el cáncer de piel con un nivel en un nivel comparable al de los dermatólogos. Los dispositivos móviles equipados con redes neuronales profundas, tiene la misma potencialidad de diagnosticar fuera de una clínica, al igual que un dermatólogo. El posicionamiento de un teléfono inteligente para el diagnóstico de lesiones cutáneas, en un futuro permitirán el acceso universal a este tipo de aplicaciones, lo cual abarata el costo de un diagnóstico sobre lesiones cutáneas y anticipará al cáncer. El aprendizaje profundo de las redes neuronales a imágenes de la piel, se podrá adaptar a otras especialidades, incluyendo oftalmología, otorrinolaringología, radiología y patología.

SkinCare [31] es una aplicación que asiste al cuidado de la piel a las personas con espina bífida (SB), ya que son susceptibles a lesiones cutáneas y problemas de la piel (ulceras crónicas, infecciones y heridas), el propósito de Skin Care es apoyar al auto cuidado de la piel, es decir de heridas dirigido por un médico. Incluye recordatorios para realizar controles diarios de la piel, así como la capacidad de informar sobre lesiones cutáneas utilizando una combinación de imágenes y descripciones de la piel, que son enviados a un portal médico.

Se ha desarrollado un sensor UV portátil que ayuda a personas ciegas [30] a medir el índice UV para el cuidado de la piel, con una aplicación móvil en el sistema operativo móvil Android. Mediante el sensor acumula datos de intensidad UV, lo cual es enviado al Smartphone a través de bluetooth, mediante aplicación es capaz de llevar información a personas normales y ciegas a través de alertas de voz, el algoritmo calcula el incidente de energía acumulada sobre la piel del usuario por unidad de área UV= $\mathrm{t}=0$ 25-UVI(t)dt[m J/m2].

\section{Discusión}

El presente análisis menciona que el uso de aplicaciones móviles para el cuidado de la piel ha permitido realizar diagnósticos en zonas remotas con el uso de la tele dermatología [32]. Es importante tener en cuenta que unas de las principales causas de 
las enfermedades permanentes de la piel en los seres humanos es la falta de protección y un diagnostico oportuno, para su posterior cuidado. A partir de la revisión sistemática se puede concluir que existen trabajos limitados que permitan realizar el cuidado de la piel mediante una aplicación móvil, utilizando sistemas inteligentes, como tal. En este estudio existió tres trabajos que integran una aplicación móvil con sistemas inteligentes que permitan el cuidado de la piel, lesiones cutáneas, etc.

Descubrimientos recientes demuestran que el uso redes neuronales profundas [5] utilizan la inteligencia artificial como un sistema inteligente para clasificar lesiones cutáneas de la piel, lo cual identifica el cáncer de piel, los diagnósticos son acertados al igual que un profesional dermatólogo. Este tipo de sistemas inteligentes permitirá abaratar costos para el diagnóstico de posibles lesiones de la piel, de esta manera se tendrá una evaluación correcta para el cuidado de la piel lo cual anticipará al cáncer.

En la obra de SkinCare [31] se evidencia una aplicación móvil en el proceso del cuidado de la, son casi desconocidas y poco accesibles por las personas. Cabe mencionar que esta aplicación ayuda al cuidado de la piel y evitar lesiones cutáneas de las personas con espina bífida, ya que es un proceso sistemático asistido por médicos, los resultados han sido favorables y menos incomodas para los usuarios.

Para finalizar en la obra de un sensor UV [30], se habla de una aplicación que ayudan a personas ciegas a partir de alertas de voz indicando la intensidad de los rayos ultra violetas, el usuario puede ingresar el tipo de piel y recibir alertas personalizadas nuestra aplicación es capaz de llevar información a personas normales y ciegas a través de alertas de voz.

\subsection{Limitaciones del estudio}

Es de vital importancia mencionar que gran parte de la literatura mencionada en las bases de datos bibliográficas no identifica claramente las palabras clave o los términos de indexación, por lo tanto él y, en consecuencia, la manera de búsqueda y su posterior metaanálisis, no relacionan a los trabajos que aportan desarrollo innovación del campo estudiado.

En este artículo hemos analizado el efecto que una característica adicional puede tener en el modelo de red propuesto con poblaciones heterogéneas, es decir, neuronas con diferentes probabilidades de reconocimiento de firmas, las cuales generan diferentes dinámicas colectivas en la red. Las simulaciones realizadas muestran que la existencia de poblaciones heterogéneas incrementa las propiedades dinámicas de la red. Además, muestran que la red presenta capacidades de codificación adicional y 
mecanismos para adaptar la estrategia de procesamiento a situaciones diferentes. Los resultados reportados sugieren que la mayoría de modelos requieren determinar los beneficios que el procesamiento de información, basado en el reconocimiento de firmas neuronales específicas, puede proporcionar a una red neuronal, por ejemplo, para ponderar la salida de una neurona, basada en el reconocimiento de firmas específicas y/o en el contenido del resto de la señal (mecanismo de contextualización), que en un momento dado emiten firmas funcionales diferentes en la salida pueden realizar tareas específicas por neurona.

\section{Conclusiones}

La aplicación de diferentes tecnologías ha permitido el cuidado de la piel, podemos mencionar la tele dermatología, se ha convertido en un servicio de salud, el cual realiza un diagnóstico dermatológico en zonas rurales para personas que sufren diferentes patologías en la piel y su posterior cuidado [17]. Sin embargo, el desconocimiento de este tipo de tecnologías, no ha permitido su adaptación e implementación en los centros de salud u hogares de las personas con problemas dermatológicos.

De otra forma, se evidencio que pocos estudios consiguen la combinación de tecnologías, es decir de aplicaciones móviles que permitan el cuidado de piel mediante reconocimiento facial aplicando sistemas inteligentes, o su vez es muy poco el número de trabajos que ayuden al cuidado de la piel, antes de contraer una patología, siendo el cáncer de piel, melanoma, y la diabetes los comunes y con alto grado de porcentaje de detección en los pacientes.

Para finalizar, una contribución al progreso de este estudio, se orientaría al desarrollo e implementación de una aplicación móvil que permita el cuidado incorporando el reconocimiento facial aplicado sistemas inteligentes como algoritmos, métodos o técnicas de inteligencia artificial y redes neuronales, el cual haga un diagnóstico y puede definir los tratamientos a seguir para contrarrestar las patologías.

\section{Agradecimientos}

El presente manuscrito se desarrolló en el contexto del Proyecto "Análisis de aplicaciones móviles que ayuden al cuidado de la piel mediante reconocimiento facial utilizando sistemas inteligentes", financiado por la Unidad de Gestión de Tecnologías -Universidad de las Fuerzas Armadas ESPE. Los autores expresan su agradecimiento a la Universidad y su instancia UGI, Unidad de Gestión de la Investigación de la UGT- 
Unidad de Gestión de Tecnologías. Nuestra gratitud a los profesores PhD. Patricio Rolando Reyes, Jose Luis Carrillo Medina, Homero Patricio Espinel Orbea tutores de Investigación, por la revisión y corrección del manuscrito

\section{References}

[1] Fombona, J., Pascual, MA., Madeira, F. Augmented reality, an evolution of the application of mobile devices. Revista de Medios y Educación, 41, pp.197-210, 2012.

[2] Phongtraychack, A., Dolgaya, D. Evolution of Mobile Applications. MATEC Web of Conference, 155, 2017.

[3] Gillenwater, T. Evolution of the smartphone Evolution of Mobile Applications. Microwave Journal, 60, pp 40-52, 2017.

[4] Othman, M., Halil, NM., Yusof, MM., Mohamed, R., Abdullah, MHA. Empowering selfmanagement through M- Health applications. MATEC Web of Conferences, 150, 2017.

[5] Esteva, A., Kuprel, B., Novoa, R.A., (...), Blau, H.M., Thrun, S. Dermatologist-level classification of skin cancer with deep neural networks. Nature, 542, pp 115-118, 2017.

[6] Cruz, R., Lopez, G. Framework para aplicaciones educativas móviles (m-learning): un enfoque tecnológico-educativo para escenarios de aprendizaje basados en dispositivos móviles. 2007.

[7] Pai, R.R., Alathur, S. Assessing mobile health applications with twitter analytics. International Journal of Medical Informatics, 113, pp 72-84,2018.

[8] Pérez-Gandía, C., García-Sáez, G., Subías, D., (...), Rigla, M., Hernando, M.E. Decision support in diabetes care: the challenge of supporting patients in their daily lives using a mobile glucose predictor. Journal of Diabetes Science and Technology, 12 , pp 243-250, 2018.

[9] Dugas, M., Crowley, K., Gao, GG, (...), Kruglanski, AW, Steinle, N. Individual differences in mode of regulation moderate the effectiveness of a mHealth pilot trial for diabetes management among older veterans. PLoS ONEOpen Access, 13

[10] Gillenwater, T., H. D. Dynamical principles in neuroscience. Reviews of Modern Physics, 78, pp. 1213-1265, 2006.

[11] Szücs, A., Pinto, R. D., Rabinovich, M. I., Abarbanel, H. D. and Selverston, A. I. Synaptic modulation of the interspike interval signatures of bursting pyloric neurons. J Neurophysiol, 89, pp. 1363-1377, 2003. 
[12] Szücs, A., Abarbanel, H. D., Rabinovich, M. I. and Selverston, A. I. Dopamine modulation of spike dynamics in bursting neurons. Eur. J Neurosci, 21(3), pp. 763772, 2005.

[13] Latorre, R., Rodriguez, F. B., Varona, P. Effect of individual spiking activity on rhythm generation of Central Pattern Generators, Neurocomputing 58-60, pp. 535--540, 2004.

[14] Latorre, R., Rodriguez, F. B., Varona, P. Neural signatures: multiple coding in spikingbursting cells, Biol. Cybern., 95, pp. 169--183, 2006.

[15] Latorre, R., Rodriguez, F. B., Varona, P. Reaction to neural signatures through excitatory synapses in Central Pattern Generator models. Neurocomputing, 70, pp. 1797--1801, 2007.

[16] Tristán, A., Rodríguez, F.B., Serrano, E. and Varona, P. Networks of neurons that emit and recognize signatures, Neurocomputing, 58-60, pp. 41--46, 2004.

[17] Carrillo-Medina, J.L. and Latorre, R. Neural dynamics based on the recognition of neural fingerprints, Front. Comput. Neurosci., 9, pp. 33, 2015. DOI: 10.3389/fncom.2015.00033.

[18] Latorre, R., Rodriguez, F. B. and Varona, P. Signature neural networks: definition and application to multidimensional sorting problems, IEEE Trans Neural Netw., 22, pp. 8--23, 2011.

[19] Watts, D.J. and Strogatz, S.H. Collective dynamics of 'small-world' networks. Nature 393, pp. 440--442. 1998, DOI:10.1038/30918.

[20] Lago-Fernández, L. F., Huerta, R., Corbacho, F., and Sigüenza, J. A. Fast response and temporal coherent oscillations in small-world networks. Phys. Rev. Lett. 84, pp. 2758--2761. 2000, DOI: 10.1103/PhysRevLett.84.2758.

[21] Carrillo-Medina, J. L. and Latorre, R. Influence of the refractory period on neural networks based on the recognition of neural signatures. In 2015 International Joint Conference on Neural Networks (IJCNN), pp. 1-9, 2015, DOI: 10.1109/IJCNN.2015.7280332.

[22] Rabinovich, M. I., Volkovskii, A., Lecanda, P., Huerta, R., Abarbanel, H.D. and Laurent, G. Dynamical encoding by networks of competing neuron groups: winnerless competition, Phys. Rev. Lett. 87(6), 068102, 2001.

[23] Afraimovich, V. S., Rabinovich, M. I. and Varona, P. Heteroclinic contours in neural ensembles and the winnerless competition principle, International Journal of Bifurcation and Chaos, 14, pp. 1195--1208, 2004.

[24] Bialek, W., Rieke, F., De Ruyter van Steveninck R. R., and Warland, D. Reading a neural code. Science, 252(5014), pp. 1854-1857, 1991. 
[25] Middleton J. W., Yu, N., Longtin, A. and Maler, L. Routing the low of sensory signals using plastic responses to bursts and isolated spikes: Experiment and theory. The Journal of Neuroscience, 31(7), pp. 2461-2473, 2011.

[26] Rieke F., Warland, D., van Steveninck, R. D. R., and Bialek, W. Spikes: exploring the neural code. The MIT Press, 1999.

[27] Kayser C., Montemurro M. A., Logothetis N. K. and Panzeri S. Spike-phase coding boosts and stabilizes information carried by spatial and temporal spike patterns, Neuron, 61(4), pp.597-608, 2009.

[28] Panzeri S., Brunel N., Logothetis N. K. and Kayser C. Sensory neural codes using multiplexed temporal scales. Trends in Neurosciences, 33(3), pp. 111-120, 2010.

[29] Baroni F., Torres J. J. and Varona P. History-dependent excitability as a single-cell substrate of transient memory for information discrimination. PLoS One, 5(12), pp. e15023, 2010.

[30] Ninell J. Dedios Mimbela, Lorena Rubio Rubio, Judith Calle Jiménez, Yudi Vásquez Domínguez. Aplicación de Dispositivos Móviles en la Medición de los Niveles de Radiación Ultravioleta y su Validación en el Distrito de Chulucanas Región Piura Perú.2017

[31] Narayanamurthy, V.; Padmapriya, P.; Noorasafrin, A.; Pooja, B.; Hema, K.; Firus Khan, A.Y.; Nithyakalyani, K.; Samsuri, F. Skin cancer detection using non-invasive techniques.2017

[32] Moreno-Ramírez, D., Argenziano, G. Teledermatology and mobile applications in the management of patients with skin lesions, 2017.

[33] Wolf, J.A., Moreau, J.F., Akilov, O., (...), Ho, J., Ferris, L.K. Diagnostic inaccuracy of smartphone applications for melanoma detection, 2013.

[34] Claus garbe, thomas k. Eigentler, Ulrich Keilholz, Axel Hauschild, John m. Kirkwood. Systematic Review of Medical Treatment in Melanoma: Current Status and Future Prospects, originally published online January 6, 2011

[35] Keele, S. (2007). Guidelines for performing systematic literature reviews in software engineering (Vol. 5). Technical report, Ver. 2.3 EBSE Technical Report. EBSE. 